\title{
Video Article \\ Assessment of Midline Lingual Point-Pressure Somatosensation Using Von Frey Hair Monofilaments
}

\author{
Nicole M. Etter ${ }^{1}$, Scott P. Breen ${ }^{2,3}$, Maya I. M. Alcala ${ }^{2,3}$, Gregory R. Ziegler ${ }^{3}$, John E. Hayes ${ }^{2,3}$ \\ ${ }^{1}$ Department of Communication Sciences and Disorders, College of Health and Human Development, The Pennsylvania State University \\ ${ }^{2}$ Sensory Evaluation Center, The Pennsylvania State University \\ ${ }^{3}$ Department of Food Science, College of Agricultural Sciences, The Pennsylvania State University
}

Correspondence to: John E. Hayes at jeh40@psu.edu

URL: https://www.jove.com/video/60656

DOI: doi:10.3791/60656

Keywords: Behavior, Issue 156, somatosensation, lingual, point pressure, touch, tongue, sensory

Date Published: 2/21/2020

Citation: Etter, N.M., Breen, S.P., Alcala, M.I.M., Ziegler, G.R., Hayes, J.E. Assessment of Midline Lingual Point-Pressure Somatosensation Using Von Frey Hair Monofilaments. J. Vis. Exp. (156), e60656, doi:10.3791/60656 (2020).

\section{Abstract}

Detection and discrimination threshold estimates for oral point pressure are assessed using Von Frey Hair monofilaments. Consistent with previously published protocols, threshold estimates are determined using a two-interval forced choice (2-IFC) paradigm with a three down/ one up approach. Detection threshold estimates determine the mean force in which a participant can identify the presence of pressure. During the detection threshold procedure, the participant is instructed to choose which of two sequentially presented observation intervals contained the tactile test stimulus. If the participant performs three correct detections in a row (i.e., 3 'hits'), the researcher decreases the stimulus to the next lower target force level. With one incorrect detection (a 'miss'), the researcher increases the force delivered to the next higher level. This threshold estimation approach is known as a 3-down/1-up adaptive staircase. Reponses are recorded on a paper ballot, and a participant's estimated threshold is defined as the geometric mean of five reversals. During the discrimination threshold procedure, the participant is asked to make a choice between two serially presented stimuli as to which is the "harder" or "stronger" pressure. The same scoring of 'hits', 'misses', and stopping points are used. Detection and discrimination testing for oral point pressure at tongue midline takes approximately 20 min to complete. Using these commercially-available clinical tools, individual touch sensation profiles for the midline tongue can be achieved in a relatively time and cost effective means.

\section{Video Link}

The video component of this article can be found at https://www.jove.com/video/60656/

Introduction

Any time we eat or drink, we determine the acceptability of a food based on multiple sensory percepts like taste, smell, and texture. Texture is not merely a physical property of the food; rather it arises from interactions of the food with the somatosensory (pressure and touch) system in the mouth. Flavor is the integrated percept that arises from multiple neuronal inputs, including taste, smell and oral touch ${ }^{1}$. Perception of food flavor, including pressure and texture information, is a key driver of food choice. Both common sense and data from numerous studies suggest people eat what they like ${ }^{2}$. However, in practice, this relationship between texture and food selection is more nuanced, as individuals avoid what they dislike ${ }^{3}$. Food selection is a behavior based on both cognitive choices and prior sensory experiences. Individual differences in smell and taste have a demonstrated ability to influence ingestive behavior ${ }^{4}$ with large variation across individuals to the point of influencing chronic diet choices ${ }^{5}$. Thus, two people may eat the same food but react very differently to it in regard to the degree of liking of that food. However, the degree to which this food preference is driven by individual differences in oral somatosensory function, including tactile and texture appreciation, remains understudied. In fact, the influences and mechanisms of oral point pressure and texture perception are much less understood relative to other oral sensory systems. Recent data suggests there may be important individual differences in oral tactile sensation abilities ${ }^{6,7,8}$. Because oral touch information is internalized and individual in nature ${ }^{9}$, it could drive individual preferences and influence food choices.

Measurements of peripheral nerve function through cutaneous pressure assessments require activation of slowly and rapidly adapting mechanoreceptors in the skin, including Merkel cells, Meissner corpuscles, Ruffini corpuscles and Pacinian corpuscles - with a high representation of slowly adapting mechanoreceptors in the face, lips, and oral mucosa that are responsive to pressure and slow stretch ${ }^{10,11}$. One relatively time and cost effective means of assessing oral tactile sensation is through the use of point-pressure assessments using Von Frey Hair (VFH) monofilaments. VFH monofilaments are widely used to assess peripheral nerve function through point pressure detection across the body, but specifically in glabrous (non-hairy) skin, including fingertip, hands, and feet ${ }^{12,13,14,15,16}$. In fact, testing with VFH monofilaments has demonstrated high test-retest reliability in the lips, tongue, and feet in healthy young, aging, and disordered populations ${ }^{16,17}$.

Assessing oral point-pressure detection and discrimination is potentially one part of a full assessment of a person's individual oral touch profile. Better understanding of individual oral touch responses could inform food choice preferences in healthy and disordered populations. A complete assessment of individual oral touch and texture perception could allow clinicians to improve recommendations for healthy aging adults as well as people with specialized (dysphagia) diets to meet their individual nutrition needs necessary for health and wellness. Select clinical populations 
with dysphagia, as well as typically aging adults, may require altered food textures to achieve adequate and safe nutrition; however, these clinical populations may also reject foods based on texture and mouth feel preferences ${ }^{18,19}$. Better understanding of the mechanisms underpinning food preferences that drive food choices, eating behavior, and diet compliance may provide novel targets for intervention, both at a systems level and an individual level.

The purpose of this assessment protocol is to characterize individual differences in oral point pressure (OPP) sensitivity by establishing detection and discrimination threshold estimates at midline tongue. This protocol uses Von Frey Hair monofilaments, commercially-available devices, to complete a relatively cost and time efficient assessment. Quantitative assessments of oral point pressure somatosensation using Von Frey hair monofilaments and this protocol were recently shown to be reliable in a cohort of healthy, young adults ${ }^{17}$ for lateral edge of the lips and tongue as a prelude to future work in speech and speech disorders. However, the present protocol and recent work has focused on midline tongue due to its involvement with manipulating foods for tasting and safe and efficient swallowing ${ }^{20}$.

\section{Protocol}

All procedures were approved by the Institutional Review Board for conducting human research at The Pennsylvania State University and were consistent with the Declaration of Helsinki.

\section{Set-up}

1. Display all monofilaments with target points between $0.008 \mathrm{~g}$ (lowest factory setting) and $15 \mathrm{~g}$ (a priori ceiling determined during pilot testing).

1. Set out the monofilaments with target forces of $0.008,0.02,0.04,0.07,0.16,0.4,0.6,1.0,1.4,2.0,4.0,6.0,8.0,10.0$, and $15.0 \mathrm{~g}$.

2. Set the monofilaments out on the table so each target level can be easily read by the experimenter.

3. Seat the participant in a comfortable chair with a glass of water within arm's reach.

4. Instruct the participant that when it is time, they will be asked to close their eyes and stick out their tongue.

1. Identify the approximate testing location at midline tongue. The testing location should be approximately $10 \mathrm{~mm}$ posterior to the tongue tip.

2. Encourage the participant to stop regularly and take a sip of water approximately every 5-10 trials or after the first incorrect response for either testing protocol, detection or discrimination.

CAUTION: The tongue should gently protrude from mouth such that the tongue tip meets the inferior edge of the lower lip. The participant should not be straining to stick their tongue out as far as possible or it will fatigue quickly and could alter testing results.

\section{Detection threshold estimates}

1. Instruct participant, "For this test, you will hear me say 'Trial 1' and 'Trial 2'. You will feel a point of pressure in one of these trials. If you think you feel a point of pressure in trial 1, put up 1 finger. If you think you feel a point of pressure in trial 2, put up 2 fingers. If you're not sure, make your best guess. Pressure will always be applied in one of the two trials."

2. Identify the starting point for testing: Determine the starting point for all participants for the detection threshold estimates. Published protocol begins at $1.0 \mathrm{~g}$. Researchers strongly suggest the experimenter begins at a suprathreshold level to be sure that the majority of participants can easily identify the first target force.

3. Tell participant to close their eyes and start the trials

1. Say Trial 1 and pause. Say Trial 2 and pause. Be sure to deliver the pressure in one of these trials such that the monofilament is pressed to the tongue surface until the monofilament buckles. Hold for a second and release.

1. Use a random number generator to create a series of 1 or 2 . Follow this series to randomly present the stimulus in Trial 1 or Trial 2 (See Supplementary Files $3 \&$ 4). Do not follow a pattern.

2. Be deliberate and intentional when applying the pressure such that the stimulus is always clearly delivered in Trial 1 or Trial 2 with a 1-s pause before and after when the Trial is said and when the stimulus is delivered.

4. Record the participant's response (See Supplementary Files 1-4 for examples)

5. Continue testing using a 3-down/1-up decision rule to select the next stimulus.

1. Move up to the next monofilament in the series after any incorrect or "missed" response.

2. Ensure that the participant gets three correct responses in a row for the experimenter to move down to the next monofilament in the series.

6. Stopping point

1. Stop testing when a participant has reached his/her stopping point. This is defined as the point when a participant has crossed over or received the test stimulus from the same target monofilament a total of five times.

2. If the participant reaches the lowest available testing target $(0.008 \mathrm{~g})$, continue delivering this target for 5 consecutive sets before stopping.

3. If the participant reaches the highest testing target $(15 \mathrm{~g})$ and cannot correctly identify the target trial, discontinue testing.

7. Estimate approximately $5-10 \mathrm{~min}$ to complete testing to determine a detection threshold estimate for midline tongue. NOTE: Stopping points apply regardless of whether the participant gets that last trial correct or incorrect. 


\section{Discrimination Threshold Estimates}

1. To complete discrimination threshold testing, follow a similar protocol to detection threshold testing.

2. Instruct participant, "For this test, you will still hear me say 'Trial 1 ' and 'Trial 2 ' but this time you will feel a point of pressure in both trials. I want you to identify which trial contained the stronger or harder point of pressure. If you think the harder/stronger pressure was in trial 1 , put up 1 finger. If you think you the harder/stronger pressure was in trial 2, put up 2 fingers. If you're not sure, make your best guess."

3. Identify a starting point

1. Begin test at three monofilament levels above the stopping point for their estimated detection threshold. Use the target stimulus and the monofilament immediately below it in force as the two discrimination stimuli.

2. Follow this example: If the participant reached a stopping point of $0.008 \mathrm{~g}$, start his/her discrimination testing at $0.07 \mathrm{~g}$ as the target stimulus and the monofilament directly below it $(0.04 \mathrm{~g})$ as the second stimulus delivered.

4. Tell the participant to close their eyes and start the trials.

1. Deliver the two stimuli after clearly stating Trial 1 or Trial 2. Again, this should be randomized, to avoid a pattern.

5. Record the participant's response (See Supplementary Files 1-4 for examples).

6. Continue testing using the same 3-down/1-up and stopping point as used in the protocol for Detection Thresholds.

1. Continue testing with $0.008 \mathrm{~g}$ with no stimulus delivered in the second trial if the participant reaches $0.008 \mathrm{~g}$ during discrimination testing (lowest manufactured level).

2. Offer the participant a drink of water if they miss two target stimuli in a row.

7. Estimate approximately $5-10 \mathrm{~min}$ to complete a discrimination threshold estimate for midline tongue.

\section{Scoring}

1. Record all target stimuli and participant response data from threshold estimate testing.

1. Record the stopping point for each individual for both detection and discrimination

2. Record the number of trials it took for the participant to successfully complete testing

3. Record the participant's threshold average by finding the first time the participant was given the eventual stopping point stimulus. Add all the target stimuli delivered between the first instance of the stopping point target force and the last and divide by the total number of trials. This gives a threshold estimate average that takes in to account the variability within a participant.

2. Set up data recording sheets using one of the two suggested options. Find examples of completed sheets using the same values can be found in the Supplementary Materials. The first option (Supplementary Files 1 \& 2) provides a data sheet and completed example in which the participant's responses and threshold estimate can be easily visualized and recorded on one sheet. The second option (Supplementary Files 3 \& 4) provides a data sheet with randomized order for presenting the test stimuli. A separate sheet is needed for each test - detection or discrimination.

\section{Cleaning equipment}

1. Do not close the monofilaments during testing or immediately following testing until they are cleaned.

2. Place the nylon end of the monofilament between an alcohol prep pad ( $70 \%$ alcohol) and gently squeeze the alcohol pad together while pulling the monofilament through.

3. Leave the monofilament in the open position, repeat the cleaning process on each monofilament used.

4. Allow the monofilaments to air dry in the open position.

5. Close the monofilaments and store them in a clean and dry environment, away from direct sunlight, as described in the manufacturer's directions.

\section{Representative Results}

When interpreting the results, it is important to remember that lower threshold estimates indicate greater sensory acuity. The use of a forced choice procedure helps separates criterion response bias (i.e., a willingness to say yes) from underlying sensitivity of the system. A lower threshold indicates an individual can perceive a lighter stimulus than those with a higher threshold estimate. Higher threshold estimates indicate decreased sensory acuity. A higher threshold estimate indicates an individual requires a larger input to cognitively perceive the stimulus. Higher threshold estimates could be due to difficulty in cognitively completing the task ${ }^{16,21}$ or due to peripheral damage associated with advancing age or disorder to the cutaneous surface of the tongue or peripheral nerves ${ }^{22}$.

Data is still being collected to further develop normative data from a sample of nominally healthy adults. However, a sample of data on individual differences in oral somatosensory function was recently published ${ }^{7}$. Combining previously published data with additional data, a total of 111 healthy participants (34M:77F; mean 32.1 years, range 18 to 68 years) completed at least one portion of the detection and/or discrimination threshold estimate protocol described here. 


\begin{tabular}{|l|l|l|l|l|}
\hline & \multicolumn{2}{|l|}{ Detection } & \multicolumn{2}{l|}{ Discrimination } \\
\hline & \multicolumn{2}{|l|}{$(\mathbf{n}=\mathbf{5 1})$} & ( $\mathbf{n}=\mathbf{1 0 7})$ \\
\hline Location & $\mathbf{M}$ (SD) & Range & M (SD) & Range \\
\hline Midline Tongue & $0.0157(0.022)$ & $0.008-0.16$ & $0.600(1.812)$ & $0.02-15$ \\
\hline
\end{tabular}

Table 1: Detection and discrimination threshold estimates for pressure point sensitivity on midline tongue. The geometric mean, standard deviation and range are provided from our representative sample. Fifty one participants completed detection threshold estimate testing (10M:41F, mean 37.1 years). A total of 107 participants completed discrimination threshold estimate testing (31M: 76F, mean 31.9 years).

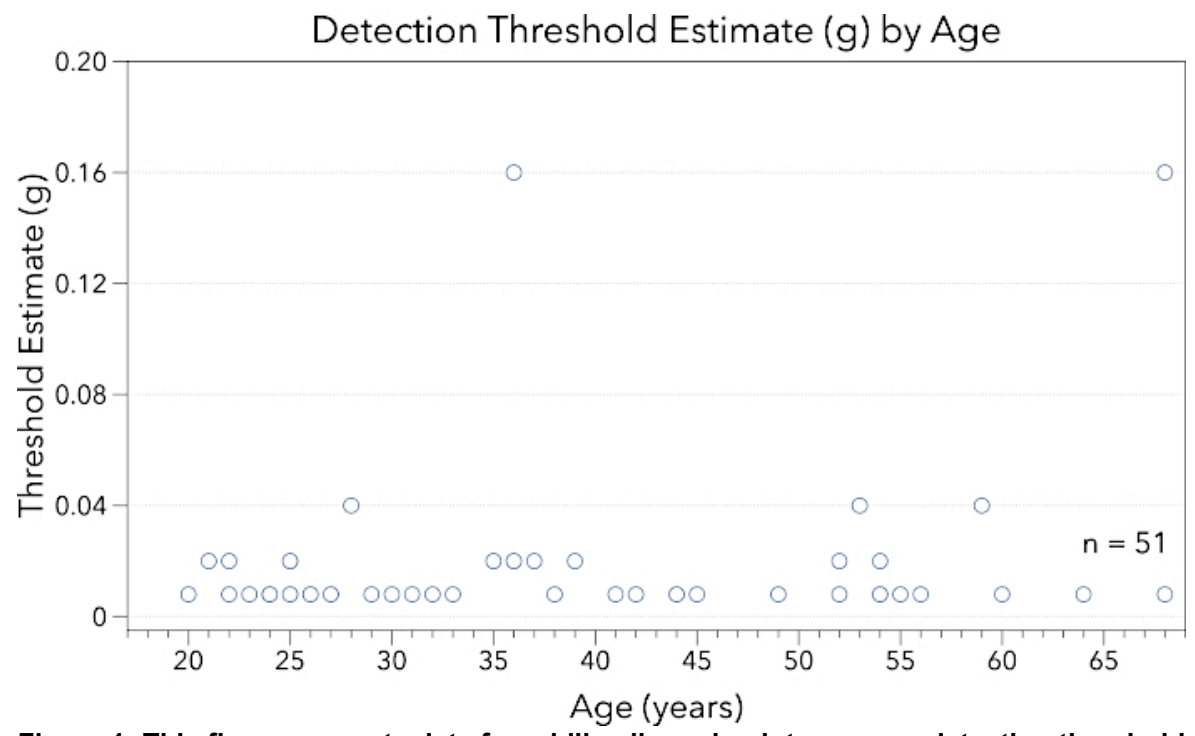

Figure 1: This figure presents data for midline lingual point pressure detection threshold estimate (g) by participant age (years). Note the $y$-axis ranges from $0.0-0.25 \mathrm{~g}$. Participants across this healthy age span demonstrated low thresholds for point-pressure detection. Please click here to view a larger version of this figure.

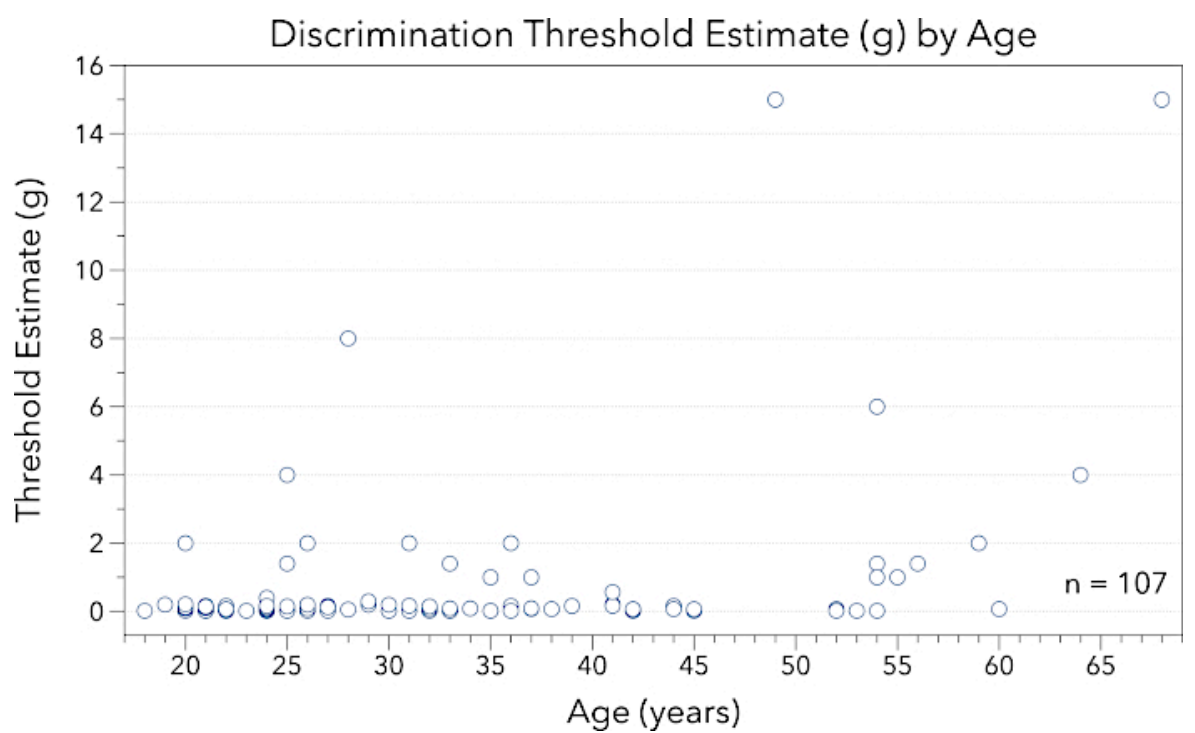

Figure 2: This figure provides data for midline lingual point pressure discrimination threshold estimate (g) by participant age (years). Note the $y$-axis ranges from $0.0-16.0 \mathrm{~g}$. Participants across this healthy age span demonstrated increased variability in discrimination threshold estimates as compared to detection threshold estimates. Please click here to view a larger version of this figure. 


\section{Supplementary Materials}

Two potential options for setting up data recording sheets are available in the Supplementary Files. Data can easily be recorded and visualized using a one page data collection sheet (Supplementary files $1 \& 2$ ). On this sheet, both detection and discrimination data can be recorded in their respective sections. The list of Von Frey Hair monofilament targets in grams $(\mathrm{g})$ and the color of the pen are included on the left hand side. An asterisk $\left(^{*}\right)$ has been placed next to the $1.000 \mathrm{~g}$ target in the 'Detection' section to remind testers of the starting point for testing using this protocol. The trial numbers are listed across the bottom to easily identify the total number of trials needed. The authors suggest following the key listed on the bottom right to mark correct and incorrect participant responses. A second data recording option is provided in Supplementary Files 3 \& 4. The series number is noted in the column marked \#. The target or test stimuli is recorded in grams in the column labeled 'Target (g)'. The next two columns are labeled 'Trial 1' and 'Trial 2' to indicate in which the trial the tester should deliver the test stimuli. This pattern was created using a random number generator. Experimenters will present the test stimuli in the trial with the "X" in it. For example, in the first series, the target stimuli would be delivered in the second trial because the " $\mathrm{X}$ " is under the Trial 2 heading. Finally, in the last column, the experimenter can record the participant's response as correct $(\mathrm{Y})$ or incorrect $(\mathrm{N})$. This column can also be used to note the number of presentations of that target to aide in identifying the stopping point.

Supplementary File 1. Please click here to view this file.

Supplementary File 2. Please click here to view this file.

Supplementary File 3. Please click here to view this file.

Supplementary File 4. Please click here to view this file.

\section{Discussion}

In previous studies, researchers observed that periodic rewetting of the tongue was an important step. For example, participants who did not regularly rewet the tongue demonstrated poorer sensory acuity. While no attempts were made to systematically determine the optimal rewetting interval, experience suggests experimenters should ask participants to rewet the tongue every few trials by bringing the tongue back into the mouth and/or having a small sip of water. Although work by Verrillo and colleagues found that vibrotactile detection thresholds were not impacted by skin hydration, these studies were completed on the hand, forearm, and cheek - not the tongue ${ }^{23}$. Because the tongue is consistently bathed in saliva, the change from wet to dry could alter sensory acuity. In fact, in work assessing salivary production and flavor appreciation in older adults, researchers found a relationship between decreased salivation and perception of umami flavor ${ }^{24}$. Aside from lingual moisture, changes in point pressure detection and discrimination threshold estimates can be impacted by a number of individual factors, including advancing $a^{22}$ or changes in cognition ${ }^{25}$ which may impact attention to task. Additionally, some previous studies have either excluded smokers or asked participants to refrain from smoking for a period of time prior to testing ${ }^{26}$.

A blindfold was not used in this protocol as prior experience suggested participants find it distracting to be blindfolded with their mouths open and tongue out. In pilot testing of the protocol, participants were startled by the stimuli or repeatedly told researchers they couldn't concentrate. Therefore, participants were simply told to close their eyes. If a participant opened their eyes during a set of stimuli presentations, that set can be repeated. Other studies in the field have chosen to use a blindfold ${ }^{20}$; however, use of a blindfold may not significantly change the results when comparing across studies ${ }^{11}$.

During initial trials of this protocol, researchers selected a ceiling of $15.0 \mathrm{~g}$. Although available monofilaments go up to $300 \mathrm{~g}$, a ceiling was selected a priori to prevent the risk of damaging the skin. Additionally, when testing an area like the tongue for which there is no internal skeletal structure, application of monofilaments higher than $15 \mathrm{~g}$ were found to move the entire tongue muscle which could activate sensory nerve endings in surrounding locations ${ }^{17}$. In the representative data sample, many healthy young adults were able to sense the $0.008 \mathrm{~g}$ target monofilament - the lowest manufactured Von Frey hair monofilament. Alternatively, Cochet-Bonnet contact aesthesiometers have been used to measure sensitivity of the cornea. It is possible this device may provide an opportunity to test at levels less than $0.008 \mathrm{~g}$; however, there are unresolved questions related to calibration of Cochet-Bonnet devices and ability to share findings across studies ${ }^{27}$.

This protocol is adapted from recent work by Etter and colleagues ${ }^{17}$ with a few key differences. In Etter's original protocol, threshold estimates were not averaged across trials. This may have missed some of the variability in participant responses. Additionally, Etter's original protocol was focused on using the tongue for speech-related movements, and therefore assessed point pressure of tongue at the right and left lateral edges just posterior to tongue tip versus midline tongue locations that may be more important for texture appreciation and swallowing ${ }^{28,29}$.

Future applications of this testing protocol may include studies of individuals with a variety of central and/or peripheral nervous system damage, as well as those with damage to the oral mucosa either from trauma, tumors, or post radiation therapy. For example, researchers recently evaluated individuals post-stroke who were experiencing oral dysphagia and noted increase thresholds, or decreased sensation in people with stroke as compared to healthy controls ${ }^{30}$. Additionally, in a review paper by Kaplan and colleagues, patients with xerostomia for a variety of medical reasons reported changes in mastication and swallowing, decreased salivary flow rates, and changes to oral mucosa ${ }^{31}$. Future work assessing the impact of these changes on tactile point pressure assessments would be beneficial to the field.

Finally, using this protocol does not require any mark to be made to the tongue to ensure exact testing location is repeated with each trial. This was originally done to increase clinical application of this protocol to medical and outpatient clinic locations. Reliability testing by Etter colleagues demonstrated high test-retest results without marking the tongue ${ }^{17}$. However, it is possible to mark the tongue using a dye to ensure a consistent target location as illustrated in a recent study by Santagiuliana and colleagues ${ }^{26}$. The appropriateness of using food dye to mark the tongue may vary depending on testing location and research question. 


\section{Disclosures}

The authors Nicole M. Etter, Scott P. Breen, and Maya I.M. Alcala have no conflicts to disclose; Breen and Alcala were students at the time the work was performed and are now employed in the food industry. The authors John E. Hayes and Gregory R. Ziegler have each received speaking or consulting fees from non-profit organizations and corporate clients in the food industry. Additionally, the Sensory Evaluation Center (Hayes, Director) at Penn State routinely conducts taste tests for the food industry to facilitate experiential learning for students. None of these organizations have had any role in study conception, design or interpretation, or the decision to publish these data.

\section{Acknowledgments}

The authors wish to thank all members of the Orofacial Physiology and Perceptual Analysis Lab (OPPAL; Etter, Director) and the Sensory Evaluation Center (Hayes, Director), both located at Penn State.

\section{References}

1. Duffy, V. B., Hayes, J. E., Bartoshuk, L. M., Snyder, D. J. Taste: Vertebrates-Psychophysics. Reference Module in Neuroscience and Biobehavioral Psychology. Elsevier (2017).

2. Tuorila, H. et al. Comparison of affective rating scales and their relationship to variables reflecting food consumption. Food Quality and Preference. 19 (1), 51-61 (2008).

3. Hayes, J. E. Measuring sensory perception in relation to consumer behavior in Rapid Sensory Profiling Techniques. in Rapid Sensory Profiling Techniques and Related Methods: Applications in New Product Development and Consumer Research. 53-69 Elsevier (2015).

4. Hayes, J. E., Feeney, E. L., Allen, A. L. Do polymorphisms in chemosensory genes matter for human ingestive behavior? Food Quality and Preference. 30 (2), 202-216 (2013).

5. Haryono, R. Y., Sprajcer, M. A., Keast, R. S. Measuring oral fatty acid thresholds, fat perception, fatty food liking, and papillae density in humans. Journal of Visualized Experiments. (88) (2014).

6. Linne, B., Simons, C. T. Quantification of Oral Roughness Perception and Comparison with Mechanism of Astringency Perception. Chemical Senses. 42 (7), 525-535 (2017).

7. Breen, S. P., Etter, N. M., Ziegler, G. R., Hayes, J. E. Oral somatosensatory acuity is related to particle size perception in chocolate. Scientific Reports. 9 (1), 7437 (2019).

8. Miles, B. L., Van Simaeys, K., Whitecotton, M., Simons, C. T. Comparative tactile sensitivity of the fingertip and apical tongue using complex and pure tactile tasks. Physiology \& Behavior. 194 515-521 (2018).

9. Bradman, M. J., Ferrini, F., Salio, C., Merighi, A. Practical mechanical threshold estimation in rodents using von Frey hairs/SemmesWeinstein monofilaments: Towards a rational method. Journal of Neuroscience Methods. 255 92-103 (2015).

10. Johansson, R. S., Trulsson, M., Olsson, K. A., Westberg, K. G. Mechanoreceptor activity from the human face and oral mucosa. Experimental Brain Research. 72 (1), 204-208 (1988).

11. Bangcuyo, R. G., Simons, C. T. Lingual tactile sensitivity: effect of age group, sex, and fungiform papillae density. Experimental Brain Research. 235 (9), 2679-2688 (2017).

12. McBride, M. R., Mistretta, C. M. Light touch thresholds in diabetic patients. Diabetes Care. 5 (3), $311-315$ (1982).

13. Moharic, M., Vidmar, G., Burger, H. Sensitivity and specificity of von Frey's hairs for the diagnosis of peripheral neuropathy in patients with type 2 diabetes mellitus. Journal of Diabetes Complications. 26 (4), 319-322 (2012).

14. Thornbury, J. M., Mistretta, C. M. Tactile sensitivity as a function of age. Journals of Gerontology. 36 (1), $34-39$ (1981).

15. Woodward, K. L. The relationship between skin compliance, age, gender, and tactile discriminative thresholds in humans. Somatosensory and Motor Research. 10 (1), 63-67 (1993).

16. Tracey, E. H., Greene, A. J., Doty, R. L. Optimizing reliability and sensitivity of Semmes-Weinstein monofilaments for establishing point tactile thresholds. Physiology \& Behavior. 105 (4), 982-986 (2012).

17. Etter, N. M., Miller, O. M., Ballard, K. J. Clinically Available Assessment Measures for Lingual and Labial Somatosensation in Healthy Adults: Normative Data and Test Reliability. American Journal of Speech-Language Pathology. 26 (3), 982-990 (2017).

18. Sura, L., Madhavan, A., Carnaby, G., Crary, M. A. Dysphagia in the elderly: management and nutritional considerations. Clinical Interventions in Aging. 7 287-298 (2012).

19. Takeuchi, K. et al. Nutritional status and dysphagia risk among community-dwelling frail older adults. Journal of Nutrition Health and Aging. 18 (4), 352-357 (2014).

20. Yackinous, C., Guinard, J. X. Relation between PROP taster status and fat perception, touch, and olfaction. Physiology \& Behavior. 72 (3), 427-437 (2001).

21. Valeriani, M., Ranghi, F., Giaquinto, S. The effects of aging on selective attention to touch: a reduced inhibitory control in elderly subjects? International Journal of Psychophysiology. 49 (1), 75-87 (2003).

22. Dunn, W. et al. Measuring change in somatosensation across the lifespan. American Journal of Occupational Therapy. 69 (3), 6903290020p1-6903290020p9 (2015).

23. Verrillo, R. T., Bolanowski, S. J., Checkosky, C. M., McGlone, F. P. Effects of hydration on tactile sensation. Somatosensory and Motor Research. 15 (2), 93-108 (1998).

24. Pushpass, R. G., Daly, B., Kelly, C., Proctor, G., Carpenter, G. H. Altered Salivary Flow, Protein Composition, and Rheology Following Taste and TRP Stimulation in Older Adults. Frontiers in Physiology. 10652 (2019).

25. Methven, L., Jimenez-Prateda, M. L., Lawlor, J. B. Sensory and consumer science methods used with older adults: a review of current methods and recommendations for the future. Food Quality and Preference. 48 333-344 (2016).

26. Santagiuliana, M. et al. Exploring variability in detection thresholds of microparticles through participant characteristics. Food \& Function. 10 (9), 5386-5397 (2019). 
27. Ehrmann, K., Saha, M., Falk, D. A novel method to stimulate mechanoreceptors and quantify their threshold values. Biomedical Physics \& Engineering Express. 4 (2), (2018).

28. Kieser, J. A. et al. The role of oral soft tissues in swallowing function: what can tongue pressure tell us? Australian Dental Journal. $\mathbf{5 9}$ (Suppl 1), 155-161 (2014).

29. Mioche, L., Hiiemae, K. M., Palmer, J. B. A postero-anterior videofluorographic study of the intra-oral management of food in man. Archives of Oral Biology. 47 (4), 267-280 (2002).

30. Schimmel, M., Voegeli, G., Duvernay, E., Leemann, B., Muller, F. Oral tactile sensitivity and masticatory performance are impaired in stroke patients. Journal of Oral Rehabilitation. 44 (3), 163-171 (2017).

31. Kaplan, I., Zuk-Paz, L., Wolff, A. Association between salivary flow rates, oral symptoms, and oral mucosal status. Oral Surgery, Oral Medicine, Oral Pathology and Oral Radiology. 106 (2), 235-241 (2008). 